\title{
Survey of Current Literature
}

This Section is intended to provide the most current phase diagram data. Guidelines for the inclusion of new information in this section are (1) systems for which no phase diagrams are given in Binary Alloy Phase Diagrams, second edition; (2) complete diagrams that are substantially different from earlier versions published in Binary Alloy Phase Diagrams, second edition, the Bulletin of Alloy Phase Diagrams, or single-topic monographs; (3) partial diagrams that alter or clarify earlier versions in the above-mentioned publications; and (4) relevant new literature of interest.

Thermodynamic consistency of the new phase diagrams was checked based on phase rules, and the diagrams were modified if necessary. However, the diagrams and texts have not gone through the ordinary reviewing process, and the final evaluations may be carried out by relevant category editors of the Alloy Phase Diagram Program. For convenience, reaction tables and crystal structure data are added when new information if available.

H. Okamoto

Literature Survey Editor

\section{Bibliography (1989-1992)}

Ag-Au 91Zhe: W. Zheng, R. Zhang, and R. Yu, "Study of the Phase Diagrams for Silver-Gold and Gold-Copper Binary Alloys at High Temperature," Chin. Sci. Bull., 36(1), $75-79$ (1991).

Ag-Cu 91Fuj: Y. Fujinaga and T. Sato, "Effect of High Pressure on the Equilibrium Phase Diagram of the $\mathrm{Ag}-\mathrm{Cu}$ System," J. Jpn. Inst. Met., 55(3), 267-271 (1991).

Ag-Ga 91Fuj: Y. Fujinaga, K. Kusaba, Y. Syono, H. Iwasaki, and T. Kikegawa, "Formation of an Intermediate Phase in Eutectic $\mathrm{Au}-\mathrm{Ge}$ and $\mathrm{Ag}-\mathrm{Ge}$ Systems under High Pressure," J. LessCommon Met, 170(2), 277-286 (1991).

Ag-Ge 91Bra: H. Bracht, N.A. Stolwijk, and H. Mehrer, "Diffusion and Solubility of Copper, Silver, and Gold in Germanium," Phys. Rev. B, Condens. Matter, 43(18), 14465-14477 (1991).

Ag-Te 91Gla: V.M. Glazov, S.G. Kim, and K.B. Nurov, "Study on Demixing of the Liquid Phase in the System Ag-Te," Zh. Fiz Khim, 65(8), 2049-2054(1991).

91Kru: E.S. Krupnikov, F. Yu. Aliev, and S.A. Aliev, "Phase Transitions in Silver Telluride," Fiz. Tverd. Tela, 33(11), 34083410 (1991).

Al-Ca 92Hoc: $\mathrm{M}$. Hoch, "The Influence of $\mathrm{Cp}(\mathrm{L}-\mathrm{s})$ on the Calculation of Phase Diagrams: The Systems Al-Ca and $\mathrm{Ca}-\mathrm{Pb}$," Calphad, 16(3), 231-241 (1992).
92Sui: H.X. Sui, M. Zhu, M. Qi, G.B.Li, and D.Z. Yang, "The Enhancement of Solid Solubility Limits of Aluminum-Cobalt Intermetallic Compounds by High-Energy Ball Milling," $J$. Appl. Phys., 71(6), 2945-2949 (1992).

Al-Co 92Ell: M. Ellner, S. Kek, and B. Predel, "On the Existence of a Phase in $\mathrm{CO}_{3} \mathrm{Al}$ of the $\mathrm{Cu}_{3} \mathrm{Au}$ Structural Type," J. Alloy. Comp., 189, 245-248 (1992).

Al-Cr 92Cos: J.G. Costa Neto, S. Gama, and C.A. Ribeiro, "Experimental Study of the Al-Cr Equilibrium Diagram," J. Alloy. Comp., 182, 271-280 (1992).

92Wen: K.Y. Wen, Y.L. Chen, and K.H. Kuo, "Crystallographic Relationships of the $\mathrm{Al}_{4} \mathrm{Cr}$ Crystalline and Quasicrystalline Phases," Metall. Trans. A, 23(9), 2437-2445 (1992).

Al-Cu 92Mit: G.B. Mitra and L. Chakravartti, "Determination of the Miscibility Gap and the Radii of Gas-Permeation Zones in Two Al-Cu Alloys from Small-Range X-Ray Scattering," $J$. Mater. Sci. Lett., II(11), 721-723 (1992).

Al-Ga 89Kre: A.A. Krebukhov and L.G. Romanov, "Equilibrium Phase Diagram of the Aluminum-Gallium System," Izv. V.U.Z Tsvetn. Metall, (6), 108-109 (1989). 